\title{
AVALIAÇÃO DA DOR EM FUNCIONÁRIOS DO SETOR DE INFORMÁTICA DE UMA INSTITUIÇÃO DE ENSINO SUPERIOR
}

\author{
PAIN ASSESSMENT IN COMPUTING STAFF OF A HIGHER EDUCATION INSTITUTION

\section{RESUMO}

A introdução do presente trabalho explana os Distúrbios Osteomusculares Relacionados ao Trabalho (DORT) são decorrentes das lesões por esforços repetitivos no ambiente de trabalho, cada vez mais discutido devido ao seu aumento. $O$ estudo se apresenta como uma pesquisa exploratória, descritiva e de campo, que teve como objetivo avaliar os sinais e sintomas de DORT em funcionários do setor de informática de uma Instituição de Ensino Superior. O estudo foi realizado no setor de informática de duas Instituições de Ensino Superior, situadas na cidade de João Pessoa - PB. Quanto aos métodos, a amostra foi do tipo não probabilística, composta por 15 funcionários da área de informática, de ambos os sexos, na faixa etária de 20 a 60 anos. A coleta de dados se deu nos meses de setembro e outubro a partir da aplicação de questionário. A pesquisa foi submetida ao comitê de ética, respeitando a resolução 466/12 do comitê de ética em pesquisa. Os resultados analíticos foram apresentados através de três tabelas e um gráfico, seguindo um processo simultâneo, facilitando assim o trabalho da análise descritiva e quantitativa. Como conclusão, o estudo mostrou que o sexo feminino foi o mais acometido pelos sintomas de DORT. Foi observado que há prevalência em determinados membros do corpo no que se refere à localização do desconforto, tendo maior prevalência na região lombar, seguidos de punho e região torácica.

Palavras-chave: Dor. Informática. Saúde do trabalhador.

\section{ABSTRACT}

Introduction of work-Related Musculoskeletal Disorders (DORS) are causes of repetitive work practices in the workplace, increasingly being debated by their

1 Graduada em Fisiotearapia pela Associação Paraibana de Ensino Renovado (ASPER). E-mail: marcelakaline2010@hotmail.com 2 Doutora em Sociologia pela Universidade Federal da Paraíba (UFPB). Docente da Associação Paraibana de Ensino Renovado (ASPER).E-mail: aliceanamenezes@yahoo.com.br 
growth. The study is presented as an exploratory, descriptive and field research that aimed to evaluate the symptoms of DORT in employees of the computer sector of a Higher Education Institution. The studies were carried out in the computer sector of two Higher Education Institutions. Higher Education, located in the city of João Pessoa - PB. About methods, the sample was of the probabilistic type, composed of 15 informatics employees, of both sexes, in the age group of 20 to 60 years. Data collection originated in the months of September and October of questionnaire applications. The consultation was submitted to the ethics committee, in compliance with resolution $466 / 12$ of the research ethics committee. The results were analyzed through three pages and a graph, following a simultaneous process, thus facilitating the work of the descriptive and quantitative analysis. In conclusion, the study showed that females were more affected by DORT symptoms. This report is there not report in the member of region, go to report in the member of region, go to region of discomfort;

Keywords: Pain. Computing. Worker's health.

\section{INTRODUÇÃO}

Distúrbios Osteomusculares Relacionados ao Trabalho (DORT) são desordens ocasionadas pela excessiva quantidade de movimentos exercida sobre um determinado músculo ou grupo muscular no qual o mesmo esteja inserido, tendo a ausência de repouso e a fraqueza muscular como fatores que predispõe ao seu processo de lesão (OLIVEIRA, et. al, 2015).

Estes tipos de distúrbios resultam em prejuízos a músculos, tendões, nervos, ligamentos, tanto dos membros superiores quanto inferiores, mais comuns nos membros superiores, caracterizados por sinais e sintomas como: dor (localizada, irradiada, generalizada), dormência, fadiga, edema, sensação de peso.

Esses sintomas podem ser "mascarados" pelo uso de análgicos e antiinflamatórios (PEREIRA et al, 2017), no qual o diagnóstico de DORT é guiado pelo profissional de saúde mediante as queixas dos sintomas relatados pelo paciente, podendo ser diagnosticado como: tenossinovites, sinovites, síndrome do túnel do carpo entre outros. Os fatores que cooperam para a intensificação de dores localizadas consistem em tarefas com movimentos demasiadamente repetitivos e posturas ergonomicamente erradas na hora de realizá-las. (SANTO; AMARANTE, 2018).

Na medida em que os graus vão aumentando, a saúde do trabalhador vai ficando cada vez mais comprometida e as possibilidades de recuperação dos membros afetados tornam-se mais complexos, podendo ser verificados desde o grau 1: Leve, não chegando a intervir em seu rendimento e nem no afastamento da função; grau 2: Moderada, não chegando a afetar seu desempenho por ser 
tolerável e nem deslocamento do setor, mais quando exige-se um pouco mais de esforço prejudica seu rendimento; grau 3: Intensa, a produtividade por esse trabalhador já sofre uma queda, tornando-o muitas vezes incapaz sua volta ao trabalho; grau 4: Insuportável: a volta ao trabalho torna-se impossível pela invalidez, como também nas atividades do cotidiano, sendo afastado de vez da função (WAGNER, 2014). Com isso, observa-se que, com o avanço das tecnologias, há maior agilidade no processo de trabalho, em contrapartida, leva o trabalhador a movimentos repetitivos e rigorosos associados a posturas erradas em busca de maior rendimento (FERREIRA, et. al, 2014). Assim, o objetivo desse estudo foi avaliar um problema presente no cotidiano de grande parte das pessoas que trabalham em ambientes no qual sua carga horária é realizada na repetitividade de afazeres sem descanso adequado.

\section{METODOLOGIA}

Esse estudo é uma pesquisa de campo descritivo, de caráter quantitativo, exploratório, no qual o pesquisador observa e relata o caso sem sua intervenção, utilizando técnicas específicas para sua coleta de dados. A pesquisa foi realizada na Faculdade Paraibana (FAP) e Associação Paraibana de Ensino Renovado (Asper) na cidade de João Pessoa. O período de aplicação foi entre os meses de setembro e outubro nos períodos de tarde e noite. A amostra foi do tipo não tipo não probabilística, composto por 15 profissionais na área de informática, de ambos os sexos, com idade entre 20 e 60 anos, com carga horária de 8h diárias, que trabalham na Faculdade Paraibana (FAP) e Associação Paraibana de Ensino Renovado (Asper). Foram excluídos da pesquisa funcionários que não trabalhavam com digitação, pessoas que não responderam de forma integral todas as perguntas do questionário sociodemográfico e tempo de trabalho inferior a 1 (um) ano.

A pesquisa foi submetida ao comitê de ética e pesquisa do Instituto Federal de Educação, Ciência e Tecnologia da Paraíba e foi aprovado sob o parecer consubstanciado de número 2.891.387. Os participantes voluntários foram esclarecidos quanto à proposta do estudo, através da leitura do Termo de Consentimento Livre e Esclarecido - TCLE e da assinatura feita por eles.

O instrumento utilizado durante a pesquisa foi um questionário elaborado pela pesquisadora, contendo 09 (nove) perguntas objetivas, sendo três com marcas de subjetividade, abrangendo aspectos sociodemográficos e informações importantes no que diz respeito aos sinais e sintomas relacionados aos distúrbios osteomusculares decorrentes das lesões por esforço repetitivo no ambiente de trabalho que causam DORT.

Para a utilização na medição da dor foi utilizada a Escala de EVA, que tem a finalidade de quantificar a dor nos membros atingidos consequentemente pelos DORT, no qual pode ser mensurada através de uma margem de 0 a 10 , onde o 0 
significa ausência de dor 1-2 grau leve, 3-7 grau moderada e de 8-10 grau intensa, solicitando aos funcionários a indicação para dor (MOURA; BRANDÃO; BARCESSAT, 2018).

Seguindo aspectos éticos, a aplicação do questionário foi em local apropriado, individualmente e de acordo com a disponibilidade de cada funcionário. Depois de preenchidos os termos de consentimento livre e esclarecido, o instrumento da coleta de dados foi entregue, preenchido e depositado em envelope, garantido o anonimato de suas respostas, como também não houve divulgação de fotos ou qualquer outro meio no qual pudesse gerar desconforto ou exposição, sendo utilizados apenas com finalidade de pesquisas científicas a nível acadêmico, conforme as normas para a realização de pesquisas envolvendo seres humanos, atendendo os critérios da Bioética do Conselho Nacional de Saúde na sua Resolução 466/12 (BRASIL, 2012).

No que tange a presente pesquisa, há risco mínimo de constrangimento e, caso ocorresse, seriam tomadas as providências, como: suspensão da aplicação e caso necessário, encaminhamento para um profissional de psicologia. Não haveria custos para o pesquisado e o mesmo seria acompanhado até a resolução total do problema.

Após análise das respostas, os funcionários participaram de uma palestra proporcionando melhor conhecimento sobre recursos fisioterapêuticos no que diz respeito a DORT, levando-os a refletir na forma como podem desempenhar melhor suas funções profissionais sem maiores prejuízos à sua saúde. O acesso à necessidade de práticas fisioterapêuticas se fez importante em prol da inibição de manifestação de DORT, como também a conscientização de cuidados com a saúde, dentro do ambiente de trabalho.

\section{RESULTADOS E DISCUSSÃO}

Para melhor compreensão, os dados coletados foram organizados através de tabelas e gráfico. Na obtenção dos resultados apresentados, foi utilizado na análise descritiva, a média e desvio padrão. A tabela 1 traz informações sobre sexo, idade e IMC dos pesquisados.

Tabela 1: Dados sociodemográficos e antropométricos da amostra.

\begin{tabular}{ccccc}
\hline \multirow{2}{*}{ Sexo } & VARIÁVEIS & N & $(\%)$ & Média \pm Desvio Padrão \\
& Homens & 2 & 13,4 & - \\
& Mulheres & 13 & 86,6 & - \\
\hline \multirow{3}{*}{ Idade } & $20-29$ & 4 & 26,7 & $67,25 \pm 17,42$ \\
& $30-39$ & 8 & 53,3 & $78,18 \pm 13,49$ \\
& $>40$ & 3 & 20 & $72,33 \pm 4,93$ \\
\hline
\end{tabular}




\begin{tabular}{ccccc}
\hline & Normal & 3 & 20 & $22,08 \pm 22,8$ \\
IMC Sobrepeso & 6 & 40 & $27,21 \pm 27,21$ \\
Obesidade I & 6 & 40 & $32,32 \pm 32,32$ \\
Obesidade II & 0 & 0 & - \\
Obesidade III & 0 & 0 & - \\
\hline
\end{tabular}

Fonte: Dados do pesquisador, 2018

A tabela 1 mostra os dados sociodemográficos e antropométricos da amostra. Em relação ao sexo houve uma prevalência do sexo feminino com $86,6 \%$ $(n=13)$. A faixa etária predominante foi de 30-39 anos $(n=8)$. Em relação ao peso corporal, visto a partir do cálculo do IMC, $20 \%(n=3)$ a amostra estava dentro do padrão de normalidade e $40 \%(n=6)$ estava acima do peso, sendo sugestivo de obesidade grau l em $40 \%$ ( $n=6)$.

De acordo com Moraes; Bastos (2017), o sexo feminino sofre mais acometimentos de DORT em relação ao sexo masculino, por passarem muito tempo digitando, em condições de trabalho insatisfatório. Isso pode acontecer por possuírem menores números de fibras musculares, pouca eficácia de reserva e conversão de glicogênio em energia, atuando na maioria das vezes, em funções de baixa qualificação, num ritmo fatigante, além das obrigações familiares que culminam em uma terceira jornada de trabalho, levando-as ao excesso de esforço, tornando-as mais propensas aos acidentes de trabalho (GRIESANG; POHL, 2017).

A faixa etária de maior incidência de DORT acontece entre os 3049 anos, em que estes trabalhadores estão economicamente eficientes, produtivos e hábeis profissionalmente (NEGRI, et al, 2014).

Os funcionários de informática, por passarem muito tempo em posição estática (sentados), digitando suas tarefas no computador, estão ficando sedentários e com o IMC em sobrepeso e obesidade grau 1. Desse modo, não desenvolvem uma atividade física, cuja consequência é a sobrecarrega dos alicerces osteomusculares (ROCHA, 2018). Os que apresentavam o peso ideal podem ser pelo constante hábito de atividades físicas (MEIRA; SILVA JÚNIOR, 2017).

Na tabela 2 encontram-se informações referentes às atividades desenvolvidas pelos funcionários, mais especificamente sobre o tempo de função, digitação e intervalo para descanso. 
Tabela 2: Dados referentes às atividades

\begin{tabular}{|c|c|c|c|}
\hline VARIÁVEIS & $\mathrm{N}$ & (\%) & Média \pm Desvia Padrão \\
\hline \multicolumn{4}{|l|}{ TEMPO NA FUNÇÃO } \\
\hline$<1$ ano & 0 & 0 & \\
\hline$>1$ ano & 15 & $100 \%$ & \\
\hline \multicolumn{4}{|l|}{ TEMPO DE DIGITAÇÃO } \\
\hline $30 \mathrm{~min}-59 \mathrm{~min}$ & 2 & 13,4 & $30 \pm 0$ \\
\hline $60 \mathrm{~min}-120 \mathrm{~min}$ & 5 & 33,3 & $60 \pm 0$ \\
\hline$>120 \mathrm{~min}$ & 8 & 53,3 & $120 \pm 0$ \\
\hline \multicolumn{4}{|l|}{ TEMPO DE INTERVALO } \\
\hline$<10 \min$ & 1 & 6,6 & $10 \pm 0$ \\
\hline Entre 10 e $20 \mathrm{~min}$ & 2 & 13,4 & $15 \pm 7,071$ \\
\hline$>20 \min$ & 0 & 0 & $x$ \\
\hline $60 \mathrm{~min}$ & 11 & 73,4 & $60 \pm 0$ \\
\hline $120 \mathrm{~min}$ & 1 & 6,6 & $120 \pm 0$ \\
\hline
\end{tabular}

Fonte: Dados do pesquisador, 2018

Com base nos resultados da tabela 2, do que foi demonstrado na tabela acima, observou-se que $100 \%(n=15)$ trabalha nesta função a mais de 1 ano. Em relação ao tempo de digitação, 2 funcionários $(13,4 \%)$ passam de 30 a 59 minutos por dia. 5 funcionários (33,3\%) digitam em média de 60 a 120 minutos por dia. 8 funcionários $(53,3 \%)$ mais que 120 minutos de tempo digitando por dia. Algumas das principais consequências advindas do uso exagerado do computador, no caso da digitação, são: tendinite, lombalgia, bucite, e síndrome do túnel do carpo.

\section{INSTRUMENTOS E PROCEDIMENTO DE COLETAS DE DADOS}

A princípio, o projeto de pesquisa foi submetido ao comitê de ética e pesquisa. Após a aprovação, os participantes voluntários foram esclarecidos quanto à proposta do estudo, através da leitura do Termo de Consentimento Livre e Esclarecido - TCLE e da assinatura feita por eles. O instrumento a ser utilizado durante a pesquisa foi um questionário elaborado pelos pesquisadores, contendo 10 (dez) perguntas objetivas, abrangendo aspectos sociodemográficos e informações importantes no que diz respeito aos sinais e sintomas relacionados aos distúrbios osteomusculares decorrentes das lesões por esforço repetitivo no ambiente de trabalho que causam DORT.

O questionário é uma técnica da pesquisa que tem como propósito obter informações sobre conhecimento de opiniões, sentimentos, interesses, expectativas e situações vivenciadas (GIL, 2002). Com o uso do questionário os dados são fieis, corretos, pois não comprometem a pessoa pesquisada, visto ser a mesma realizada no anonimato, propondo mais facilidade, rapideze baixo custo ao pesquisador, além 
de traduzir os objetivos específicos da pesquisa (MERTEN, 2011). Os trabalhadores serão informados de que a pesquisa será importante para a composição de um estudo cujo intuito é ampliar o conhecimento sobre exercícios fisioterapêuticos no ambiente de trabalho. Também será dito a estes que receberão um material explicativo de orientação, proporcionando melhor conhecimento no que diz respeito aos distúrbios osteomusculares de DORT, levando-os a refletir na forma como podem desempenhar melhor suas funções profissionais. Seguindo aspectos éticos, a aplicação do questionário será em local apropriado e de acordo com a disponibilidade de cada funcionário, podendo ser aplicado no período da tarde, sendo ainda garantido o sigilo e confidencialidade das informações individuais, os dados obtidos serão utilizados apenas para finalidade de pesquisas científicas de nível acadêmico.

\section{RISCOS}

No que tange a presente pesquisa, há risco mínimo de constrangimento e, caso ocorra, serão tomadas as providências, como: suspensão da aplicação e caso necessário, encaminhamento para um profissional de psicologia. Não haverá custos para o pesquisado e o mesmo será acompanhado até a resolução total do problema. Para garantir anonimato e maior segurança por parte dos voluntários, não haverá divulgação de fotos ou qualquer outro meio que possa gerar desconforto ou exposição. A coleta de dados servirá apenas para o âmbito da pesquisa científica, sendo a mesma realizada de forma individual e em local apropriado. Depois de preenchidos os termos de consentimento livre e esclarecido, os instrumentos de coleta de dados serão depositados pelo participante em envelopes distintos, garantido o anonimato de suas respostas. Faz-se importante esclarecer que todos os custos financeiros relacionados à pesquisa serão de responsabilidade dos pesquisadores.

\section{BENEFÍCIOS}

Após análise das respostas dos participantes, em um intervalo de 3 (três) meses, haverá a devolutiva de uma cartilha de orientação a estes, proporcionando melhor conhecimento no que diz respeito aos distúrbios osteomusculares de DORT, levando-os a refletir na forma como podem desempenhar melhor suas funções profissionais. Outro benefício a que terão acesso será à importância das práticas fisioterapêuticas, que podem ser utilizadas em prol da inibição de manifestação de DORT, como também a conscientização de cuidados com a saúde, dentro do ambiente de trabalho.

Os riscos de, futuramente, possuírem DORT está relacionado não apenas ao tempo que passam digitando, mas também ao tempo de intervalo, que é mínimo. 
Os dados da tabela mostram que 1 funcionário $(6,6 \%)$ passa menos de dez minutos de descanso; 2 funcionários $(13,4 \%)$ tem entre 10 e 20 minutos de descanso, enquanto 11 funcionários $(73,4 \%)$ passam cerca de 60 minutos de descanso.

Montenegro (2018) constatou que os trabalhadores de informática com mais de 1 ano nessa função apresentaram o maior índice de DORT. Maeno (2018) afirma que o aumento de anos na função, a falta de organização do tempo, e o aumento de metas a serem cumpridas e a diminuição de pausa, são fatores que predispõem a distúrbios musculoesqueléticos, associados a fadiga, ansiedade e queda no rendimento.

Tabela 3: Localização do desconforto

\begin{tabular}{cccc}
\hline VARIÁVEIS/LOCAIS & N & (\%) & Média Desvia Padrão \\
\hline Região cervical & 1 & 6,6 & $8 \pm 9,89$ \\
Trapézio descendente & 2 & 13,3 & $8,5 \pm 9,19$ \\
Ombro & 2 & 13,3 & $8,5 \pm 9,19$ \\
Braço & 1 & 6,6 & $8 \pm 9,89$ \\
Punho & 4 & 26,6 & $9,5 \pm 7,77$ \\
Cabeça dos metacarpos & 1 & 6,6 & $8 \pm 9,89$ \\
Região torácica & 3 & 20 & $9 \pm 8,48$ \\
Lombar & 5 & 33,3 & $10 \pm 7,07$ \\
Quadril & 1 & 6,6 & $8 \pm 9,89$ \\
Panturrilha & 1 & 6,6 & $8 \pm 9,89$ \\
Tornozelo & 1 & 6,6 & $8 \pm 9,89$ \\
\hline
\end{tabular}

Fonte: Dados do pesquisador, 2018

A tabela 3 mostra os locais do corpo referidos como áreas de desconforto pelos trabalhadores. O local mais acometido foi a região lombar, com 33,3\% ( $=5)$, seguido do punho com $26,6 \%(n=4)$; Vale salientar que o tipo de dor mais presente era a moderada. A região torácica com $20 \%(n=3)$; trapézio descendente e ombro com 13,3\%(n=2) e com 6,6\% nas regiões cervical, braço, cabeça dos metacarpos, quadril, panturrilha e tornozelo. Quando indagados sobre os sinais e sintomas de DORT, tais como a sensação de peso, o cansaço, as parestesias, o edema, o formigamento, a fadiga muscular e a dor, esta última foi a que obteve prevalência, em que dos $100 \%(n=15)$ dos entrevistados, apenas $26,7 \%(n=4)$ não se referiam a nenhum tipo de desconforto.

De acordo com Falcão (2018), as partes do corpo que sofrem mais sobrecargas pelo mau posicionamento do corpo e também por adotar um período longo na posição estática são a região lombar e torácica da coluna vertebral e o punho, visto que esses músculos permanecem tensionados por longos períodos, gerando desconforto que podem estar relacionados à fadiga muscular. 
Beneli; Acosta (2017) relatam que as alterações posturais podem ser advindas da ausência de mobiliários ergonomicamente corretos, que contribuem para os vícios posturais, principalmente membros superiores, coluna cervical e lombar, como também a diminuição na qualidade de vida, resultando em funcionários altamente estressados, cansados mentalmente e fisicamente, devido à grande exigência de suas tarefas.

Gomes; Barbosa; Perfeito (2018) afirmam que a dor, ao movimentar especificamente algum membro afetado, sensação de peso, formigamento, ausência de sensibilidade, perda de força, edema local, surgem como os primeiros sintomas de DORT e quando diagnosticado precocemente e logo em seguida feito às intervenções necessárias, terá grandes chances de diminuição e melhora desses sintomas.

Avalie-se o gráfico a seguir:

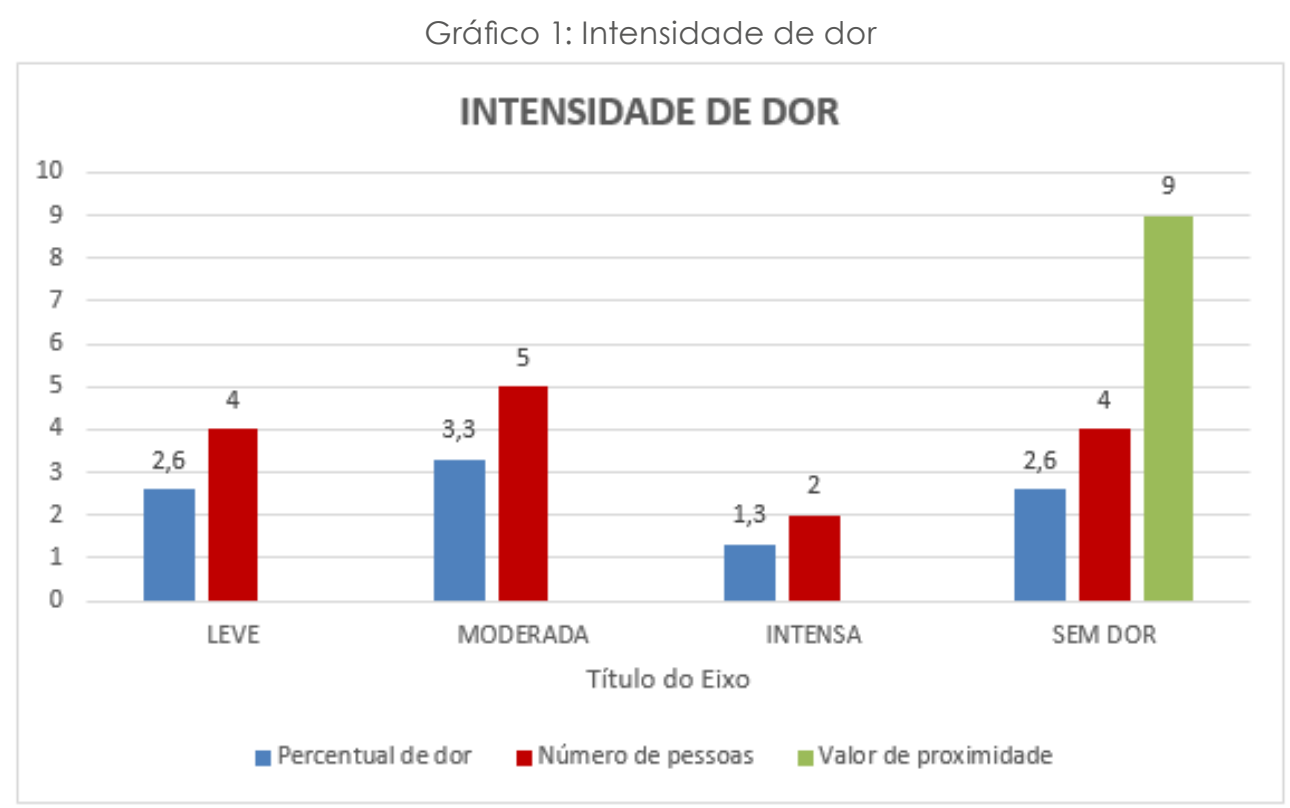

Fonte: Dados do pesquisador, 2018

No gráfico 1, observa-se a intensidade de dor baseado na Escala de EVA, com as seguintes classificação: leve $2,6 \%(n=4)$, moderada $3,3 \%(n=5)$, intensa $1,3 \%$ $(n=2)$ e sem dor $2,6 \%(n=4)$, no qual a maior prevalência se dá na intensidade de dor moderada atingindo o maior número de funcionários. Algumas medidas eram tomadas pelos participantes a partir do quadro álgico, dentre elas: uso de analgésicos, uso de imobilizador, uso de gelo, uso de substâncias e outros não precisavam fazer uso de tais medidas. Quanto a fisioterapia, relataram não procurar.

É preciso atentar para as diferenças entre as regiões acometidas pela intensidade da dor. As regiões do corpo mais acometidas pelo mau uso do computador são os membros superiores devido à repetitividade dos movimentos.

\section{INTER SCIENTIA}

REVISTA INTERSCIENTIA | V. 7 | N. 2 | P. 67-79 | JUL-DEZ/2019 
A coluna vertebral pode ser lesionada pela má postura, caso a cadeira não esteja nas condições necessárias a sua fisiologia; a falta de irrigação sanguínea nos membros inferiores, por passar a maior parte do tempo sentado, além do estresse, dores nas costas e a síndrome da visão do computador, este último ocasionado por uso ininterrupto, normalmente por mais de duas horas (SILVEIRA, 2012; SAKAMOTO, 2014).

Assim, trabalhadores que utilizavam o computador como instrumento para as tarefas do dia-a-dia e demonstrados pela Escala Analógica Visual (EVA), apresentam constantes dores musculoesqueléticas chegando ser de moderada a severa, afetando negativamente seu rendimento no trabalho, tornando-os até mesmo incapacitados para tais atividades antes desenvolvidas com o melhor do seu potencial (MENEZES, 2017).

A partir dos dados obtidos por Longen et.al (2018), a Escala de EVA demonstrou que os trabalhadores, cujo relato foi possuir dor moderada, poderiam ter limitada a execução de seus movimentos, o que pode levar à perda de força muscular. Em contrapartida, é necessário tomar os cuidados com o tempo de execução de tarefas e com as pausas para se ter uma boa saúde.

O estudo mostrou que o sexo feminino foi o mais acometido pelos sintomas de DORT. Foi observado que há prevalência em determinados membros do corpo no que se refere à localização do desconforto, tendo maior prevalência na região lombar, seguidos de punho e região torácica.

\section{CONSIDERAÇÕES FINAIS}

Com a busca por agilidade e soluções rápidas as instituições, IES, investem cada dia mais em novas tecnologias, em que a maioria das funções desenvolvidas por trabalhadores, principalmente em setores de informática, exige movimentos repetitivos.

Quanto às propostas analíticas e as limitações, para averiguação dos dados coletados, delimitamos um tempo para análise e construção de tabelas e gráficos para melhor organização na expressão dos resultados obtidos através do questionário sociodemográfico. Os dados foram analisados por meio de estatística descritiva, ajudando os pesquisadores a encontrarem e divulgarem novos dados sobre os distúrbios osteomusculares de DORT e seu tratamento, por meio de estruturação flexível para melhor compreensão do fenômeno estudado. Para tais feitos, usamos a técnica de categorização para os dados retirados dos questionários, objetivando facilitar o trabalho da análise descritiva, onde foram utilizados recursos estatísticos básicos para os dados quantitativos, visando fortalecer a pesquisa através de dados reais.

Com base no que foi construído, verificou-se que é de extrema importância tratar da temática de sinais e sintomas de DORT na informática, devido ao 
crescimento de acometimento nesses funcionários, permitindo ainda que através dessa pesquisa, novos estudos venham a ser realizados, uma vez que contribuem não apenas para a identificação dos distúrbios osteomusculares relacionados ao trabalho, mas, sobretudo no modo como preveni-los. Destacando que as palavras contidas nesse estudo não são as últimas, mas estão em um conjunto de outros estudos, e ao mesmo tempo pode servir de base para outras pesquisas.

\section{REFERÊNCIAS}

BENELI, L.M.; ACOSTA B.F. Efeitos de um programa de ginástica laboral sobre a incidência de dor em funcionários de uma empresa de software. Revista Saúde e Meio Ambiente-RESMA, Três Lagoas, v. 4, n.1, p. 66-76, jan./jul. 2017.

BRASIL. Conselho Nacional de Saúde. Resolução $n^{\circ}$ 466, de 12 de dezembro de 2012. Aprova normas regulamentadoras de pesquisas envolvendo seres humanos. Brasília: Diário Oficial da União, 2013.

FALCÃO, T.J.M. Uma ferramenta de apoio a análise de risco biomecânico de trabalhadores em ambiente informatizado.2018. 111f. Programa de Pós-graduação. Dissertação (mestre em Modelagem computacional do conhecimento). Universidade Federal de Alagoas (UFAL), Maceió, 2018.

FERREIRA, E.S.C, et al-Análise Ergonômica de Ambientes de Trabalho Informatizados na Região do Cariri. Revista Interfaces: Saúde, Humanas e Tecnologia, Faculdade Leão Sampaio- Juazeiro do Norte-CE, v. 2, n. 6,2014.

GOMES, J.M.; BARBOSA, D.S; PERFEITO.R.S. Identificação e ocorrência de LER/ DORT em profissionais da saúde. Revista Carioca de Educação Física, v. 13, n. 1, p. 62-76. ISSN 2177-6482, 2018.

GRIESANG, C.; POHL, H.H. As LER/DORT na visão do trabalhador adoecido: um estudo de caso. IN: ALVES, L. M. SCHMIDT, S. B. F. K. (Org.) Saúde do trabalhador: realidades, intervenções e possibilidades no Sistema Único de Saúde. p. 15-32,EDUNISC. Santa Cruz do Sul-RS, 2017.

LONGEN, W.C, et al. Avaliação da incapacidade e qualidade de vida de trabalhadores da produção de indústrias cerâmicas. Núcleo de Promoção e Atenção Clínica à Saúde do Trabalhador (NUPAC) da UNESC - Criciúma (SC), Brasil.

DOI: 10.5327/Z16794435201801 13. Rev Bras Med Trab. 2018; 16(1): 10-8. 
MAENO, M. Perícia ou imperícia. Laudos da justiça do trabalho sobre LER/ DORT. 2018.400f.Programa de Pós-graduação. Tese (doutorado em saúde pública) Universidade de São Paulo- Faculdade de Saúde Pública. São Paulo, 2018.

MEIRA, P.R.S.; JUNIOR SILVA, J.A. Qualidade de vida no serviço público o caso dos servidores efetivos do Senado Federal. COLÓQUIO-Revista do Desenvolvimento Regional- Faccat-Taquara/RS-v. 14, n.2, julh.dez/2017.

MENEZES, F.S. A influência das condições psicofisiológicas individuais na produtividade laboral de funcionários de uma instituição de ensino superior. 2017.143f. Programa de Pós-graduação (Engenheira de Produção). Universidade de Tecnológica Federal do Paraná. Ponta Grossa-PR, 2017.

MONTENEGRO, B.M.G. Avaliação da carga mental e incidência de sintomas osteomusculares em profissionais de tecnologia da informação. 2018.56f. Programa de Pós-graduação (Especialista em Higiene Ocupacional). Instituto Federal de Educação, Ciência e Tecnologia da Paraíba-Campus Patos-PB. Patos, 2018.

MORAES, P.W.T.; BASTOS, A.V.B. Os sintomas de LER/DORT: Um estudo comparativo entre bancários com e sem diagnostico. Psicologia: Ciência e Profissão, Jul/Set.2017, v.37, n³, p.624-637. https://doi.org/10.1590/1982 3703001862016.

MOURA, J.P.G.; BRANDÃO, L.B.; BORCESSAT, A.R.P. Estudo da Terapia Fotodinâmica (PDT) no reparo de lesões teciduais: estudo de casos clínicos. Estação Científica (UNIFAP)https://periodicos.unifap.br/index.php/estacao.ISSN 2179-1902 Macapá, v. 8, n. 1, p. 103-110, jan./abr. 2018.

NEGRI,J.R, et al. Perfil sociodemográfico e ocupacional de trabalhadores com LER/ DORT: Estudo Epidemiológico. Revista Baiana de Saúde Pública, Piracicaba-SP, v.38, n.3, p.555-570, jul/set.2014.

OLIVEIRA, M.M, et al. Problema crônico de coluna e diagnóstico de distúrbios osteomusculares relacionados ao trabalho (DORT) autorreferidos no Brasil: Pesquisa nacional de saúde, Epidemiol.Serv.Saúde, Brasília, v.24, n.2, p.287-296, abr-jun.2015.

PEREIRA, G.C. A, et al. Ocorrência dos sinais e sintomas de DORT na equipe de enfermagem. Brazilian Journal of Surgery and Clinical Research-BJSCR (ISSN online: 2317-4404).vol.18, n 3, p.58-67(Mar-Mai 217), Minas Gerais-BH.

ROCHA, L.F. Avaliação de Riscos Biomecânicos na Saúde Ocupacional por meio de imagens infravermelhas. 2018. 132f.Programa de Pós-graduação. Dissertação (mestre em Ciências). Universidade Tecnológica do Paraná, Curitiba, 2018. 
SANTO, P.E.; AMARANTE, M.S. Prevenção de doenças do trabalho por lesões por esforços repetitivos (LER) ou distúrbios osteomusculares relacionados ao trabalho (DORT) em uma produção com final de linha manual. Pesquisa e Ação, v.4, n.1, p.28-34. ISSN 2447-0627, maio, 2018.

SAKAMOTO, F.S. Analise ergonômica dentro de um setor de Tecnologia de Informação. 30 f. Monografia da Especialização Universidade Tecnológica Federal do Paraná. Curitiba, 2014.

SILVEIRA.M.M;PASQUALOTTI.A;COLUSSI.E.L. Envelhecimento e usuários de informática: repercussões de um programa ergonômico. Estud.Interdicipl, Porto Alegre, v.19,n.1,p.219-234, 2014.

WAGNER, José Luis.Cartilha Sobre LER/DORT. Rio Grande do Sul, 2014. 